\title{
OS CROMOSSOMOS DOS ANFÍBIOS ANUROS DO GÊNERO Eleutherodactylus (Anura: Leptodactylidae: Eleutherodactylinae)
}

\section{THE CHROMOSOMES OF THE AMPHIBIAN ANURANS OF THE GENUS Eleutherodactylus (Anura: Leptodactylidae: Eleutherodactylinae)}

\author{
João Reinaldo da Cruz de Campos $^{{ }^{*}}$, Sanae Kasahara ${ }^{1}$ \\ 1* Autor para contato: Universidade Estadual Paulista - UNESP, Departamento de \\ Biologia Celular e Molecular, Instituto de Biociências, Rio Claro, SP, Brasil; \\ (19) 35264150 e (19) 3526 4156; e-mail: jrcc@rc.unesp.br
}

Recebido para a publicação em 17/04/2006

Aceito para a publicação em 08/05/2006

\section{RESUMO}

O gênero Eleutherodactylus é o mais abundante entre os vertebrados, com mais de 700 espécies que se distribuem em todo continente americano, desde o sul dos Estados Unidos, México, América Central e, na América do Sul, até o norte da Argentina. Até a presente data, relativamente poucas espécies foram cariotipadas e a maioria delas apenas com coloração convencional. Contrariando a sugestão de que os anuros possuem cariótipos bastante conservados, os Eleutherodactylus têm mostrado uma grande variação nos números diplóides que vão de $2 n=18$ até 36 e, também, diferenças no número fundamental de braços cromossômicos, de NF=32 a 58, observadas mesmo entre espécies com 2n coincidentes. Foram descritas variações intra-específicas no número de cromossomos e, além disso, cromossomos sexuais diferenciados citologicamente em quatro espécies, fato esse raro entre os anuros. No presente trabalho, é apresentada a relação das espécies de Eleutherodactylus já cariotipadas, com seus respectivos números diplóides, bem como com os números fundamentais quando disponíveis. Tais informações citogenéticas são de grande relevância, na medida que poderão contribuir para a resolução das questões de taxonomia e sistemática que cercam o referido gênero.

Palavras-chave: citogenética, bandamento cromossômico, cariótipo 


\begin{abstract}
The genus Eleutherodactylus is the most abundant among vertebrates, with its more than 700 species, which are distributed over the American continent, from the South of the United States, through Mexico, Central and South America, to the North of Argentina. To date, a relatively small number of species has been karyotyped, the great majority of them only conventionally. Contrary to the conservative karyotypic pattern characteristic of most anurans, the Eleutherodactylus species show a great variation in diploid numbers, from $2 \mathrm{n}=18$ to 36 , and also a distinct fundamental number of chromosome arms, from $\mathrm{FN}=32$ to 58, even among species sharing the same $2 \mathrm{n}$. Intraspecific variations in the number of chromosomes were described and, in addition to that, cytologically differentiated sex chromosomes in four species, an uncommon occurrence among anurans. In this paper, a survey of karyotyped species of Eleutherodactylus along with their diploid numbers, as well as the available fundamental numbers, is presented. These cytogenetic data are of relevance, as they may contribute to clarify taxonomic and systematic issues within the genus.
\end{abstract}

Key words: cytogenetics, chromosome banding, karyotype

\section{Introdução}

Entre os anuros, a família Leptodactylidae é uma das mais diversificadas, com 1243 espécies, sendo que a grande maioria delas pertence à subfamília Eleutherodactylinae (Frost, 2004). Segundo esses autores, dos 13 gêneros nela incluídos, Eleutherodactylus, com mais de 700 espécies descritas, corresponde ao gênero mais abundante entre todos os vertebrados. Os herpetólogos, contudo, estimam que esse número possa ser bem maior, acima de 1000, devido ao fato de muitas espécies novas não terem sido sequer coletadas e outras permanecerem depositadas em coleções científicas ainda sem uma descrição formal ou com erros de identificação (Castanho e Haddad, 2000, Padial e De La Riva, 2005).

As espécies de Eleutherodactylus são terrestres e, como todos os Eleutherodactylinae, apresentam desenvolvimento direto do ovo. Pelo menos duas das espécies, E. jasperi e E. coqui, têm fertilização interna, apesar de apenas a primeira delas apresentar viviparidade (Duellman e Trueb, 1994, Frost, 2004). Tal modo de reprodução, sem passar pelo estágio larval, deve ter contribuído para a ocupação de uma variedade de habitats, que vai do semiaquático ao arbóreo, bem como para uma ampla distribuição geográfica das espécies.

Representantes do gênero Eleutherodactylus são encontrados no sul dos Estados Unidos, onde foram introduzidos, México, América Central e, na América do Sul, até o norte da Argentina. Embora ocorram em quase todo o continente americano, houve uma especiação muito acentuada nas Antilhas, sendo que em algumas ilhas oceânicas Eleutherodactylus é o único gênero de Anura encontrado ou o gênero predominante em outras (Lynch, 1976, Duellman e Trueb, 1994). É importante ressaltar também a notável radiação de representantes do gênero nas ilhas do Caribe, com mais de 125 espécies conhecidas, enquanto no Norte da América do Sul e na América Central estão ainda em estágio de grande radiação e especiação (Schmid et al., 2002a).

Devido ao grande número de espécies e à variabilidade morfológica dos animais, incluindo variação no padrão de coloração, uma extensa confusão taxonômica e sistemática envolve as espécies de Eleutherodactylus. Diversas tentativas de agrupamentos foram realizadas (Lynch, 1976, Heyer, 1984, Lynch e Duellman, 1997), porém, não são conclusivas. Particularmente, em relação aos Eleutherodactylus que 
ocorrem no Sul e Sudeste do Brasil, Lynch (1976) estabeleceu os grupos de E. binotatus, E. parvus e E. lacteus, com base nos caracteres morfológicos das espécies. Posteriormente, Heyer (1984) descartou a monofilia do grupo de $E$. binotatus, com a criação do cluster de E. guentheri, no qual incluiu, entre outras, as espécies anteriormente alocadas naquele grupo morfológico. Mais tarde, Lynch e Duellman (1997), analisando a sistemática, ecologia e biogeografia de algumas espécies de Eleutherodactylus da América do Sul, desconsideraram a proposta de Heyer (1984) de monofilia do grupo de E. guentheri, de modo que as espécies que antes pertenciam ao grupo de $E$. binotatus nele permanecem até hoje. Os autores propuseram quatro outros agrupamentos para os representantes brasileiros descritos até aquela data, além dos três já descritos em Lynch (1976), mas pelo menos dez espécies, também de ocorrência no Sul e Sudeste do Brasil, não foram incluídas em nenhum dos grupos morfológicos (Heyer, 1985, Garcia, 1996, Langone e Segalla, 1996, Castanho e Haddad, 2000, Frost, 2004).

Uma característica marcante do gênero Eleutherodactylus é uma grande variabilidade cariotípica interespecífica (Bogart, 1981 e 1991, Bogart e Hedges, 1995), diferentemente do que tem sido observado entre os anuros, cujas espécies são, em geral, muito conservadas sob o ponto de vista citogenético. Desse modo, as informações obtidas a partir das análises cromossômicas poderiam contribuir, em certa extensão, para esclarecer as questões relacionadas à taxonomia e sistemática do gênero. Levando-se em consideração que o número de espécies cariotipadas de Eleutherodactylus têm aumentado nos últimos anos, sendo que as mais recentes revisões foram da década de 90 (King, 1990, Kuramoto, 1990), o presente trabalho teve como objetivo realizar uma atualização desses dados com consultas a artigos publicados, resumos de congressos e, mesmo, obtidos por comunicação pessoal.

\section{Dados Citogenéticos}

Até a presente data, o número de representantes cariotipados pertencentes ao gênero Eleutherodactylus é relativamente pequeno diante da abundância das espécies descritas, ainda que tenha aumentado nos últimos anos. As revisões de King (1990) e Kuramoto (1990) relatam os números diplóides de cerca de 60 e 70 espécies, respectivamente. De acordo com os dados por nós levantados e apresentados na Tabela 1, mais de 110 espécies foram cariotipadas desde então, algumas delas com descrição de mais de um número diplóide.

A grande maioria das espécies analisadas é proveniente da América Central e do Norte da América do Sul. Em contraste com um padrão cariotípico relativamente conservado característico da maioria dos anuros, o gênero Eleutherodactylus apresenta uma extensa variação no número diplóide, que vai de $2 \mathrm{n}=18$ a $2 \mathrm{n}=36$. A Tabela 1 mostra o número diplóide de espécies de Eleutherodactylus acompanhado do respectivo número fundamental de braços cromossômicos (NF), quando disponível, procedência e tipos de coloração diferencial, quando aplicada nos estudos citogenéticos. Com base nas informações levantadas na Tabela 1, construímos o gráfico da Figura 1, no qual pode-se observar a distribuição dos números diplóides em um total de 120 cariótipos encontrados em espécies de Eleutherodactylus.

Para a maioria das espécies, os autores não relatam o NF ou sequer apresentam o correspondente cariograma. Devido a esse fato e à dificuldade de se determinar a morfologia cromossômica pela observação de uma única figura, foram colocados na tabela somente os NF indicados pelos próprios autores (Bogart, 1970a, 1973a, Green et al., 1980, Savage e DeWeese, 1980, Miyamoto, 1983, Schmid et al., 1992, Schmid et al., 2003, Siqueira Jr et al., 2005, Campos, 2006) e os levantados por DeWeese (1975). Observa-se uma extensa variação que vai de 32 a 58, tendo sido encontrados $\mathrm{NF}=32,34,35,36,38,40$, $44,46,50,52$ e 58.

De um modo geral, os cariótipos com números cromossômicos altos têm predominância de elementos de um só braço, dos tipos telocêntricos e subtelocêntricos, enquanto aqueles com números baixos são constituídos, majoritariamente, de elementos de dois braços, dos tipos metacêntricos e submetacêntricos. Tal fato é sugestivo de que a variação do número diplóide e, portanto, a evolução cariotípica no gênero Eleutherodactylus se deve basicamente a rearranjos do tipo fusão ou fissão cêntrica. Uma observação mais detalhada dos carió- 
tipos, porém, mostra, muitas vezes, NF distintos correspondendo ao mesmo número de cromossomos, ou mesmo que não são incomuns os casos de diferenças marcantes na quantidade de heterocromatina. Desse modo, outros tipos de eventos, como translocação recíproca, inversão, ganho ou perda de heterocromatina, devem ter, certamente, contribuído para produzir a excepcional diferenciação cariotípica observada em espécies de Eleutherodactylus (Bogart, 1981, 1991, King, 1990, Schmid et al., 1992, Schmid et al., 2002a, Schmid et al., 2002c, Schmid et al., 2003, Bogart e Hedges, 1995).

As extensas mudanças cromossômicas observadas em Eleutherodactylus poderiam, segundo Schmid et al. (2003), ter se iniciado por processos de fissão em um cariótipo ancestral hipotético com $2 n=26$ e $\mathrm{NF}=52$, composto de cromossomos metacêntricos e submetacêntricos, tal como observado em muitas famílias da subordem Neobatrachia (King, 1990); a partir daí, em um cariótipo com numerosos telocêntricos, reordenamentos estruturais subseqüentes teriam produzido a grande variedade de cariótipos observada atualmente nas espécies desse gênero.

DeWeese (1975), baseando-se nos dados citogenéticos obtidos até então, preconizou dois conjuntos de espécies de Eleutherodactylus. O Caribenho corresponderia ao das espécies que apresentam valores altos de NF e número diplóide modal de 26, tendo as inversões como os principais mecanismos de reordenamento dos cariótipos. O conjunto Mesoamericano corresponderia ao das espécies cujos cariótipos, derivados basicamente de translocações robertsonianas, têm número diplóide modal de 22.

Além de grande variação interespecífica observada nos cariótipos dos Eleutherodactylus são relatados nesse gênero casos de variação intraespecífica e até mesmo intra-individual. Os números cromossômicos ímpares encontrados nas espécies $E$. bakeri $(2 \mathrm{n}=27 \mathrm{e}$ $28)$, E. cundalli $(2 \mathrm{n}=29$ e 30), E. glaucoreius $(2 \mathrm{n}=29$ e 30), E. glandulifer $(2 \mathrm{n}=31)$ e E. heminota $(2 \mathrm{n}=27$ e 28) são, de acordo com Bogart (1991), resultantes muito provavelmente de fusão cêntrica, com redução do número cromossômico. Tal rearranjo foi sugerido também por Siqueira Jr et al. (2005) para explicar a variação encontrada intra-individualmente em $E$. fenestratus ( $2 n=34$, 33 e 32).

Os números diplóides distintos mencionados para E. biporcatus (E. maussi) com $2 \mathrm{n}=20$ e $2 \mathrm{n}=35$ e 36 (DeWeese, 1975, Schmid et al., 1992, Schmid et al., 2002c, Savage e Myers, 2002), E. brandisfordii com 2n=20 e 2n=18 (Bogart, 1970a, 1970b, DeWeese,1975), E. diastemata com 2n=18 e 2n=20 (Bogart, 1970a, 1970b, León, 1970) e E. rugulosus com $2 n=20$ e $2 n=22$ (Brum-Zorrilla e Saez, 1968, DeWeese, 1975, Miyamoto, 1983) poderiam indicar espécies distintas, porém, identificadas de forma incorreta, ou, então, variação cromossômica interpopulacional.

Um achado inusitado no gênero Eleutherodactylus refere-se à presença de cromossomos sexuais diferenciados do ponto de vista citológico em quatro espécies, isto é, em E. biporcatus (E. maussi) (Schmid et al., 1992, Schmid et al., 2002c), E. euphronides e E. shrevei (Schmid et al., 2002a) e E. riveroi (Schmid et al., 2003), principalmente se levarmos em conta que os anuros mostram de um modo geral o par XY ou o ZW indistinguíveis citologicamente por coloração convencional e diferencial (Schmid et al., 1991). E. biporcatus (E. maussi) e E. riveroi constituem os primeiros casos de anfíbios com descrição de cromossomos sexuais múltiplos do tipo $\mathrm{X}_{1} \mathrm{X}_{1} \mathrm{X}_{2} \mathrm{X}_{2}$ (ou $\mathrm{XXAA}$ ): $\mathrm{X}_{1} \mathrm{X}_{2} \mathrm{Y}$ (ou $\mathrm{XAA}^{\mathrm{Y}}$ ), resultante de fusão de $\mathrm{Y}$ com um autossomo, tendo sido observado que machos com cromossomos sexuais $\mathrm{X}_{1} \mathrm{X}_{2} \mathrm{Y}$ coexistem com machos XY na mesma população de ambas as espécies. Eleutherodactylus euphronides e E. shrevei mostram sistema ZZ:ZW, no qual o cromossomo W tem a peculiaridade de ser de tamanho gigante, devido preponderantemente ao acúmulo de várias categorias de seqüências repetitivas de DNA.

Poucas espécies brasileiras foram estudadas citogeneticamente, sendo elas Eleutherodactylus binotatus, E. guentheri, E. holti, E. juipoca, E. lacteus, E. parvus e Eleutherodactylus sp., todas da região Sul e Sudeste (Beçak, 1968, Brum-Zorrilla e Saez, 1968, Beçak e Beçak, 1974, Lucca e Jim, 1974, Lucca et al., 1974, Siqueira Jr et al., 2004, Campos, 2006). Há, contudo, um breve relato para $E$. fenestratus (Siqueira Jr et al., 2005) e E. ramagii (F. Ananias, comunicação pessoal) de localidades do Norte e Nordeste, respectivamente. As espécies $E$. altamazonicus, E. conspicillatus, E. lacrimosus e E. sulcatus (Bogart, 1970a, Bogart, 1973, DeWeese, 1975), embora não tenham sido cariotipadas por 
pesquisadores brasileiros, sabe-se hoje que são também de ocorrência no Norte do Brasil, conforme levantamento realizado por Souza (2003) no estado do Acre. Embora o número de espécies brasileiras cariotipadas seja relativamente pequeno, parece que podem ser agrupadas segundo os dois conjuntos sugeridos por DeWeese (1975). De fato, todas as espécies da região Sul e Sudeste têm mostrado números diplóides mais baixos, ou seja, $2 n=20$ ou $2 n=22$, enquanto os da região Norte e Nordeste, $2 n=30$ e $2 n=34$, com exceção de E. sulcatus, que possui 22 cromossomos, (Beçak, 1968, Brum-Zorrilla e Saez, 1968, Beçak e Beçak, 1974, Lucca e Jim, 1974, Lucca et al., 1974, Bogart, 1970a, 1973, DeWeese, 1975, Siqueira Jr et al. 2004, Siqueira Jr et al. 2005, Campos, 2006).

Ainda no gênero Eleutherodactylus, é importante mencionar os dados relativos à quantidade de DNA obtidos em E. binotatus, E. guentheri e E. shrevei. Beçak e Beçak (1974) encontraram em $E$. binotatus uma quantidade de DNA nuclear cerca de quatro vezes maior do que em E. guentheri, embora ambas tenham o mesmo número cromossômico. Por outro lado, Schmid et al. (2002a) observaram em E. shrevei uma quantidade de DNA extremamente pequena, indicando um dos menores genomas entre os anfíbios e, talvez, entre os vertebrados. Isso é ainda mais surpreendente se se levar em conta que no cariótipo da referida espécie os autores estimam que só a heterocromatina C-positiva totaliza 34,7\% e 41,2\% do genoma haplóide de macho e fêmea, respectivamente, o que significa uma redução bem maior das seqüências de DNA de cópia única e moderadamente repetitiva.

\section{Considerações Finais}

Dos diversos relatos citogenéticos existentes para espécies de Eleutherodactylus, pode-se dizer que a grande maioria foi baseada quase que exclusivamente em análises com coloração convencional. O emprego de algum tipo de coloração diferencial é bastante raro e, em alguns trabalhos, se restringem ao uso do bandamento C, da marcação de Ag-RON ou de ambos (Bogart, 1981, 1991, Bogart e Hedges, 1995, Kaiser et al., 1994, Kaiser et al., 1995). É importante enfatizar, porém, que existem dados obtidos com técnicas mais resolutivas, como por exemplo, as de coloração com fluorocromos base-específicos, FISH com sondas teloméricas e de DNAr 18S e 5S (Schmid et al., 1992, Schmid et al. 2002a, Schmid et al. 2002b, Schmid et al., 2003, Siqueira Jr et al., 2004, Siqueira Jr et al., 2005, Campos, 2006).O uso de coloração diferencial tem mostrado casos de variação cromossômica em Eleutherodactylus, principalmente no que se refere à quantidade e distribuição de heterocromatina, a nível interespecífico e mesmo interpopulacional, além de informações sobre a composição molecular dessas regiões repetitivas.

Tais fatos reforçam a importância de se estender as análises cariológicas a um maior número de espécies de Eleutherodactylus com o uso de diferentes técnicas citogenéticas. Os dados assim obtidos poderão explicar a marcante diversificação cariotípica e, conseqüentemente, os possíveis mecanismos de evolução cromossômica no gênero, assim como auxiliar na resolução de questões de taxonomia e sistemática no referido grupo.

\section{Agradecimentos}

Os autores agradecem ao Programa de Capacitação de Pessoal (CAPES), ao Programa de Caracterização, Conservação e Uso Sustentável da Biodiversidade do Estado de São Paulo (BIOTAFAPESP) e ao Conselho Nacional de Desenvolvimento Científico e Tecnológico (CNPq). 
Tabela 1 - Relação das espécies de Eleutherodactylus cariotipadas até o momento, com número diplóide (2n), número fundamental (NF), procedência, tipo de coloração diferencial e referência

\begin{tabular}{|c|c|c|c|c|c|}
\hline Espécie & $2 n$ & NF & Procedência & $\begin{array}{l}\text { Coloração } \\
\text { diferencial }\end{array}$ & Referência \\
\hline E. abbotti & 18 & & & & Bogart (não publicado)* \\
\hline E. altae & 26 & 46 & & & DeWeese, 1975 \\
\hline E. altamazonicus & 34 & 36 & Peru & & $\begin{array}{l}\text { Bogart, 1970a, 1970c, DeWeese, } \\
1975\end{array}$ \\
\hline E. alticola & 32 & & Jamaica & $\mathrm{C}$ & Bogart e Hedges, 1995 \\
\hline E. amplinympha & 28 & & Caribe & & Kaiser et al., 1994 \\
\hline E. andi & 22 & 38 & & & DeWeese, 1975 \\
\hline E. andrewsi & 32 & & Jamaica & $\mathrm{C}$ & Bogart e Hedges, 1995 \\
\hline E. angelicus & 20 & 36 & Costa Rica & & Miyamoto, 1983 \\
\hline E. antillensis & 26 & 50 & Porto Rico & $\mathrm{C}$ & DeWeese, 1975, Bogart, 1981 \\
\hline E. atkinsi & 28 & & Cuba & $\mathrm{C}$ & Bogart, 1981 \\
\hline E. augusti & 22 & & & & Duellman, 1967 \\
\hline E. auriculatus & 18 & & Cuba & $\mathrm{C}$ & Bogart, 1981 \\
\hline E. bakeri & 27,28 & & Haiti & & Bogart, 1991 \\
\hline E. berkenbuschii & 22 & 36 & México & & $\begin{array}{l}\text { Savage e DeWeese, 1979, Miyamoto, } \\
1983\end{array}$ \\
\hline E. binotatus & 22 & 38 & Brasil & $\begin{array}{c}\text { C, Ag- } \\
\text { RON, F, } \\
\text { FISH }\end{array}$ & $\begin{array}{l}\text { Beçak e Beçak, 1974, Siqueira Jr et } \\
\text { al., 2004, Campos, } 2006\end{array}$ \\
\hline \multirow{2}{*}{$\begin{array}{l}\text { E. biporcatus (E. } \\
\text { maussi })\end{array}$} & 20 & 38 & & & DeWeese, 1975 \\
\hline & 35,36 & 42 & Venezuela & $\begin{array}{l}\text { C, Ag- } \\
\text { RON, F }\end{array}$ & $\begin{array}{l}\text { Schmid et al., 1992, Schmid et al., } \\
\text { 2002c, Savage e Myers, } 2002\end{array}$ \\
\hline E. bogotensis & 34 & 36 & & & $\begin{array}{l}\text { DeWeese, 1975, Bogart e Drewery } \\
\text { (não publicado)* }\end{array}$ \\
\hline \multirow{2}{*}{ E. bransfordii } & 20 & 40 & & & Bogart, 1970a, 1970b \\
\hline & 18 & 36 & & & DeWeese, 1975 \\
\hline E. brittoni & 26 & 52 & Porto Rico & $\mathrm{C}$ & DeWeese, 1975, Bogart, 1981 \\
\hline E. bufoniformis & 22 & & & & Bogart, 1973b** \\
\hline $\begin{array}{l}\text { E. } \\
\text { caryophyllaceus }\end{array}$ & 32 & 32 & Costa Rica & & DeWeese, 1975, Miyamoto, 1984 \\
\hline E. cavernicola & 30 & & Jamaica & & Bogart e Hedges, 1995 \\
\hline $\begin{array}{l}\text { E. } \\
\text { charlottevillensis }\end{array}$ & 36 & 36 & Tobago & $\begin{array}{l}\mathrm{C}, \mathrm{Ag}- \\
\mathrm{RON}\end{array}$ & Kaiser et al., 1995 \\
\hline
\end{tabular}




\begin{tabular}{|c|c|c|c|c|c|}
\hline E. conspicillatus & 34 & 36 & Peru & & Bogart, $1970^{\mathrm{a}}, 1973^{\mathrm{a}}$, DeWeese, 1975 \\
\hline E. cooki & 26 & & Porto Rico & $\mathrm{C}$ & Bogart, 1981 \\
\hline E. coqui & 26 & & Porto Rico & $\mathrm{C}$ & DeWeese, 1975, Bogart, 1981 \\
\hline E. crassidigitus & 22 & 40 & & & DeWeese, 1975 \\
\hline E. cruentus & 32 & 32 & Costa Rica & & DeWeese, 1975, Miyamoto, 1984 \\
\hline E. cundalli & 29,30 & & Jamaica & $\mathrm{C}$ & Bogart, 1991, Bogart e Hedges, 1995 \\
\hline E. cuneatus & 32 & & Cuba & $\mathrm{C}$ & Bogart, 1981 \\
\hline E. decoratus & 22 & & Costa Rica & & Savage e DeWeese, 1979,1980 \\
\hline \multirow{2}{*}{ E. diastema } & 18 & 36 & Costa Rica & & Bogart, 1970a, 1970c \\
\hline & 20 & 36 & Costa Rica & & Léon, 1970 \\
\hline E. dimidiatus & 30 & & Porto Rico & $\mathrm{C}$ & Bogart, 1981 \\
\hline E. discoidalis & 22 & & Argentina & & Brum-Zorrilla e Saez, 1968 \\
\hline E. eneidae & 26 & & Porto Rico & $\mathrm{C}$ & Bogart, 1981 \\
\hline E. escoces & 20 & 36 & Costa Rica & & Miyamoto, 1983 \\
\hline E. euphronides & 32 & & Antilhas & $\mathrm{C}, \mathrm{F}, \mathrm{FISH}$ & Schmid et al., 2002a \\
\hline E. fenestratus & $\begin{array}{l}34(33 \\
32)\end{array}$ & 34,35 & Brasil & $\begin{array}{l}\text { C, Ag- } \\
\text { RON, } \\
\text { FISH }\end{array}$ & Siqueira Jr et al., 2005 \\
\hline E. fitzingeri & 22 & 38 & Costa Rica & & eon, 1970, DeWeese, 1975 \\
\hline E. fleischmanni & 20 & 36 & Costa Rica & & $\begin{array}{l}\text { Bogart, } 1970^{\mathrm{a}}, 1973^{\mathrm{a}} \text {, DeWeese, } 1975 \text {, } \\
\text { Miyamoto, } 1983\end{array}$ \\
\hline E. fuscus & 28 & & Jamaica & $\mathrm{C}$ & Bogart e Hedges, 1995 \\
\hline E. gaigeae & 34 & & & & Bogart, $1973 b^{* *}$ \\
\hline E. glandulifer & 31 & & Haiti & & Bogart, 1991 \\
\hline E. glaucoreius & 29,30 & & Jamaica & $\mathrm{C}$ & Bogart, 1991, Bogart e Hedges, 1995 \\
\hline E. goini & 32 & & Cuba & $\mathrm{C}$ & Bogart, 1981 \\
\hline E. gossei & 26 & & Jamaica & $\mathrm{C}$ & Bogart e Hedges, 1995 \\
\hline E. grabhami & 30 & & Jamaica & & Bogart e Hedges, 1995 \\
\hline E. greggi & 22 & 40 & & & DeWeese, 1975 \\
\hline E. griphus & 32 & & Jamaica & $\mathrm{C}$ & Bogart e Hedges, 1995 \\
\hline E. gryllus & 26 & 50 & Porto Rico & & DeWeese, 1975, Bogart, 1981 \\
\hline E. guentheri & 22 & 44 & Brasil & $\begin{array}{l}\text { C, Ag- } \\
\text { RON, F, } \\
\text { FISH }\end{array}$ & $\begin{array}{l}\text { Beçak, 1968, Brum-Zorrilla e Saez, } \\
\text { 1968, Beçak e Beçak, 1974, Siqueira } \\
\text { Jr et al., 2004, Campos, } 2006\end{array}$ \\
\hline
\end{tabular}




\begin{tabular}{|c|c|c|c|c|c|}
\hline E. gulosus & 20 & & $\begin{array}{c}\text { Costa Rica } \\
\text { Panamá }\end{array}$ & & Savage e Myers, 2002 \\
\hline E. hedricki & 26 & 52 & Porto Rico & & DeWeese, 1975, Bogart, 1981 \\
\hline E. heminota & 27,28 & & Haiti & & Bogart, 1991 \\
\hline E. holti & 20 & & Brasil & & Lucca et al., 1974 \\
\hline E. hylaedactylus & 26 & & Peru & & Bogart, 1970a \\
\hline E. jamaicensis & 30 & & Jamaica & $\mathrm{C}$ & Bogart e Hedges, 1995 \\
\hline E. jasperi & 26 & & Porto Rico & & Drewry e Jones, 1976 \\
\hline \multirow{2}{*}{ E. johnstonei } & \multirow{2}{*}{28} & & Caribe & & Kaiser et al., 1994 , \\
\hline & & & Jamaica & $\mathrm{C}$ & Bogart e Hedges, 1995 \\
\hline E. juipoca & 22 & 40 & Brasil & $\begin{array}{c}\mathrm{C}, \mathrm{Ag}- \\
\mathrm{RON}, \mathrm{F}, \\
\text { FISH }\end{array}$ & Campos, 2006 \\
\hline E. junori & 24 & & Jamaica & $\mathrm{C}$ & Bogart e Hedges, 1995 \\
\hline E. karlschmidti & 30 & 40 & Porto Rico & & DeWeese, 1975, Bogart, 1981 \\
\hline E. klinikowskii & 32 & 38 & Cuba & $\mathrm{C}$ & Green et al., 1980, Bogart, 1981 \\
\hline E. lacrimosus & 34 & 36 & & & DeWeese, 1975 \\
\hline E. lacteus & 20 & 40 & Brasil & & Lucca e Jim, 1974 \\
\hline E. lanthanites & 36 & & & & Bogart, 1973b \\
\hline E. leprus & 26 & & México & & Bogart, 1973a \\
\hline E. limbatus & 32 & & Cuba & $\mathrm{C}$ & Bogart, 1981 \\
\hline E. locustus & 26 & & Porto Rico & & Bogart, 1981 \\
\hline E. longirostris & 22 & 38 & & & DeWeese, 1975 \\
\hline E. luteolus & 28 & & Jamaica & $\mathrm{C}$ & Bogart e Hedges, 1995 \\
\hline E. marnockii & 30 & & $\begin{array}{l}\text { Estados } \\
\text { Unidos }\end{array}$ & & Bogart, 1970a, 1970c, 1973a \\
\hline E. martinicensis & 28 & & Caribe & & Kaiser et al., 1994 \\
\hline E. megacephalus & 20 & & Costa Rica & & Savage e Myers, 2002 \\
\hline \multirow{2}{*}{ E. melanostictus } & \multirow{2}{*}{22} & 38 & Costa Rica & & Bogart, 1970a, 1970b, 1973a \\
\hline & & 36 & Costa Rica & & Savage e DeWeese, 1980 \\
\hline E. milesi & 22 & 44 & & & DeWeese, 1975 \\
\hline E. mimus & 20 & 36 & & & DeWeese, 1975 \\
\hline E. nitidus & 22 & & & & Duellman, 1967 \\
\hline E. nortoni & 30 & & Haiti & $\mathrm{C}$ & Bogart, 1991 \\
\hline
\end{tabular}




\begin{tabular}{|c|c|c|c|c|c|}
\hline E. orcutti & 32 & & Jamaica & $\mathrm{C}$ & Bogart e Hedges, 1995 \\
\hline E. opimus & 20 & & $\begin{array}{l}\text { Colômbia } \\
\text { Panamá }\end{array}$ & & Savage e Myers, 2002 \\
\hline E. pantoni & 26 & & Jamaica & $\mathrm{C}$ & Bogart e Hedges, 1995 \\
\hline E. parvus & 22 & & Brasil & $\begin{array}{l}\text { C, Ag- } \\
\text { RON, F, } \\
\text { FISH }\end{array}$ & $\begin{array}{l}\text { Beçak e Beçak, 1974, Siqueira Jr et } \\
\text { al., 2004, Campos, } 2006\end{array}$ \\
\hline E. patriciae & 22 & & & & Bogart (não publicado)* \\
\hline E. pentasyringos & 28 & & Jamaica & $\mathrm{C}$ & Bogart e Hedges, 1995 \\
\hline \multirow{2}{*}{$\begin{array}{l}\text { E.p.planirostris } \\
\text { (E.ricordii) } \\
\text { E.p. goinii }\end{array}$} & 32 & & $\begin{array}{l}\text { Estados } \\
\text { Unidos }\end{array}$ & & Bogart, 1973a, 1981 \\
\hline & 32 & & Cuba & $\mathrm{C}$ & Bogart, 1981 \\
\hline E. podiciferus & 18 & & Costa Rica & & $\begin{array}{l}\text { Bogart, 1970a, 1970c, 1973a, León, } \\
1970\end{array}$ \\
\hline E. portoricencis & 26 & 52 & Porto Rico & & Bogart, 1973a \\
\hline E. punctariolus & 20 & 36 & Costa Rica & & DeWeese, 1975, Miyamoto, 1983 \\
\hline E. ramagii & 30 & 30 & Brasil & & F. Ananias (com. pessoal) \\
\hline E. ranoides & 22 & & Costa Rica & & Brum-Zorrilla e Saez, 1968 \\
\hline E. rayo & 22 & 36 & Costa Rica & & Savage e DeWeese, 1979 \\
\hline E. rhodopis & 18 & 36 & & & DeWeese, 1975 \\
\hline E. richmondi & 30 & 58 & Porto Rico & $\mathrm{C}$ & DeWeese, 1975, Bogart, 1981 \\
\hline E. ridens & 34 & 36 & Costa Rica & & DeWeese, 1975, Miyamoto, 1984 \\
\hline E. riveroi & 35,36 & 36 & Venezuela & $\begin{array}{c}\text { C, Ag- } \\
\text { RON, F, } \\
\text { FISH }\end{array}$ & Schmid et al., 2003 \\
\hline E. rugosus & 20 & & $\begin{array}{l}\text { Costa Rica } \\
\text { Panamá }\end{array}$ & & Savage e Myers, 2002 \\
\hline \multirow[b]{2}{*}{ E. rugulosus } & 20 & 36 & Costa Rica & & DeWeese, 1975, Miyamoto, 1983 \\
\hline & 22 & 36 & & & $\begin{array}{l}\text { Brum-Zorrilla e Saez, 1968, } \\
\text { DeWeese, } 1975\end{array}$ \\
\hline E. shrevei & 32 & & Antilhas & $\mathrm{C}, \mathrm{F}, \mathrm{FISH}$ & Schmid et al., 2002a \\
\hline E. sisyphodemus & 32 & & Jamaica & $\mathrm{C}$ & Bogart e Hedges, 1995 \\
\hline E. sulcatus & 22 & 38 & & & DeWeese, 1975 \\
\hline E. talamancae & 22 & 36 & Costa Rica & & $\begin{array}{l}\text { DeWeese, 1975, Savage e DeWeese, } \\
1979\end{array}$ \\
\hline E. taurus & 20 & 36 & Costa Rica & & DeWeese, 1975, Miyamoto, 1983 \\
\hline
\end{tabular}




\begin{tabular}{|c|c|c|c|c|c|}
\hline $\begin{array}{l}\text { E. } \\
\text { terraeboliviaris }\end{array}$ & 36 & & Tobago & $\begin{array}{l}\text { C, Ag- } \\
\text { RON }\end{array}$ & Kaiser et al., 1995 \\
\hline E. unicolor & 30 & 36 & Porto Rico & & DeWeese, 1975, Bogart, 1981 \\
\hline E. urichi & 30 & & & & Schmid, 2002a \\
\hline E. variabilis & 34 & 36 & Peru & & Bogart, 1970a \\
\hline E. varians & 18,34 & & Cuba & $\mathrm{C}$ & Bogart, 1970a, 1981 \\
\hline $\begin{array}{l}\text { E. } \\
\text { ventrimarmoratus }\end{array}$ & 36 & 36 & Peru & & Bogart, 1970a, 1970c, 1973a \\
\hline E. vocalis & 22 & & Costa Rica & & Savage e DeWeese, 1979 \\
\hline E. wightmanae & 26 & & Porto Rico & $\mathrm{C}$ & Bogart, 1981 \\
\hline E. zugi & 32 & & Cuba & $\mathrm{C}$ & Bogart, 1981 \\
\hline $\begin{array}{l}\text { Eleutherodactylus } \\
\text { sp. } 1\end{array}$ & 26 & & Peru & & Bogart, 1970a \\
\hline $\begin{array}{l}\text { Eleutherodactylus } \\
\text { sp. } 2\end{array}$ & 22 & 40 & Brasil & $\mathrm{C}, \mathrm{Ag}, \mathrm{F}$ & Campos, 2006 \\
\hline
\end{tabular}

$\mathrm{C}=$ banda $\mathrm{C}$, Ag-RON= marcação pelo nitrato de prata, $\mathrm{F}=$ coloração por fluorocromos base-específicos, FISH= hibridação in situ com sondas fluorescentes de DNAr ou telomérica

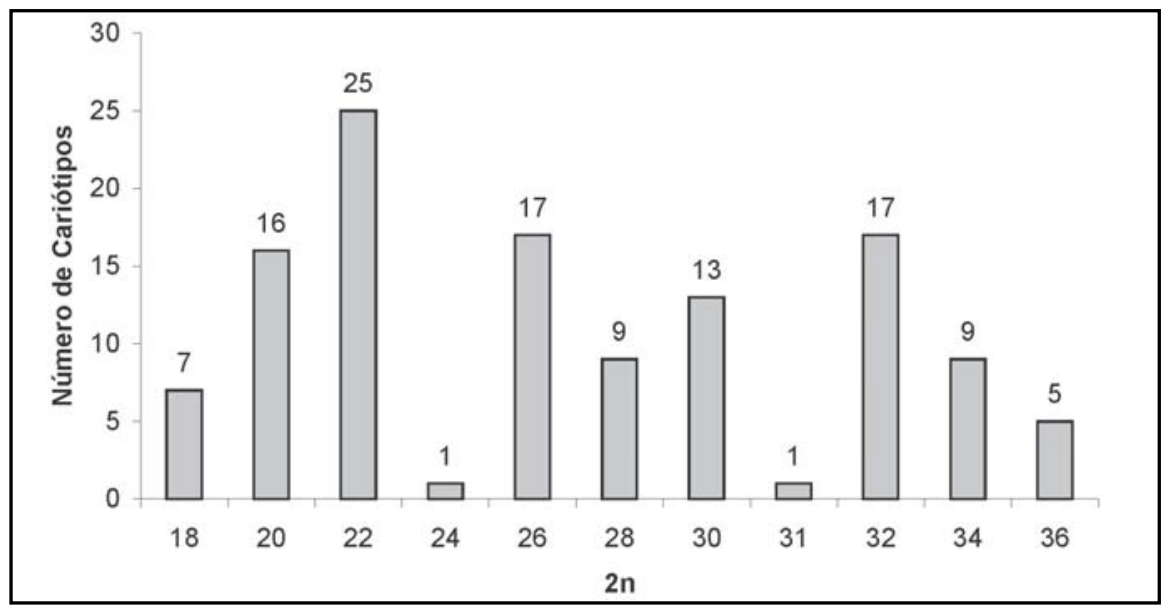

Figura 1 - Gráfico com indicação dos números diplóides observados em 120 cariótipos de espécies de Eleutherodactylus, levantados na Tabela 1

\section{REFERÊNCIAS}

1. BEÇAK, M.L. Chromosomal analysis of eighteen species of Anura. Caryologia, v.21, p.191-208, 1968.

2. BEÇAK, M.L., BEÇAK, W. Diploidization in Eleutherodactylus (Leptodactylidae-Amphibia). Experientia, v.30, p.624-625, 1974.

3. BOGART, J.P. Los cromosomas de anfibios anuros del género Eleutherodactylus. Act. IV Congr. Latin. Zool., v.1, p.65- 78,1970a.

4. BOGART, J.P. Cromosomas em algunos generos de anuros. Act. IV Congr. Latin. Zool., v.1, p.79-85,1970b.

5. BOGART, J.P. Systematic problems in the amphibian family Leptodactylidae (Anura) as indicated by karyotypic analysis. Cytogenetics, v.9, p.369-383, 1970c.

6. BOGART, J.P. Evolution of anuran karyotypes. In: VIAL, J.L. (Ed.) Evolutionary Biology of Anurans. Columbia:

Publ. UEPG Ci. Biol. Saúde, Ponta Grossa, 12 (1): 27-38, mar. 2006 
University Missouri Press, p.337-349, 1973a.

7. BOGART, J.P. 1973b apud KURAMOTO, M. A list of chromosome numbers of anuran Amphibians. Bull. Fukuoka Univ. Educ., v.39, p.83-127, 1990.

8. BOGART, J.P. Chromosomes studies in Sminthillus from Cuba and Eleutherodactylus from Cuba and Puerto Rico (Amphibia: Leptodactylidae). Life Sci. Contr. R. Ontario Mus.,v.129, p.1-22, 1981.

9. BOGART, J.P. The influence of life history on karyotypic evolution in frogs. In: GREEN, D.M., SESSIONS, S.K. (Eds.) Amphibian Cytogenetics and Evolution. New York: Academic Press, p.233-258, 1991.

10. BOGART, J.P., HEDGES, S.B. Rapid chromosome evolution in Jamaican frogs of the genus Eleutherodactylus (Leptodactylidae). J. Zool., v.235, p.9-31, 1995.

11. BRUM-ZORRILLA, N., SAEZ, F.A. Chromosomes of Leptodactylidae (Amphibia, Anura). Experientia, v.24, p.969, 1968.

12. CAMPOS, JRC. Similaridade cariotípica entre Barycholos ternetzi e cinco espécies do gênero Eleutherodactylus do sudeste do Brasil (Anura, Leptodactylidae, Eleutherodactylinae). Dissertação de Mestrado, Instituto de Biociências, UNESP, Rio Claro, SP, 2003.

13. CASTANHO, L.M., HADDAD, C.F.B. New species of Eleutherodactylus (Amphibia: Leptodactylidae) from Guaraqueçaba, Atlantic Forest of Brazil. Copeia, v.3, p.777781, 2000.

14. DeWEESE, J.E. Chromosomes in Eleutherodactylus (Anura: Leptodactylidae). Mamm. Chrom. Newsl., v.16, p.121123, 1975.

15. DREWRY, G.E. JONES, K.L. A new ovoviviparous frog, Eleutherodactylus jasperi (Amphibia, Anura, Leptodactylidae), from Puerto Rico. J. Herpetol., v.10, p.161$165,1976$.

16. DUELLMAN, W.E. Additional studies of chromosomes of anuran amphibians. Syst. Zool., v.16, p.38-43, 1967.

17. DUELLMAN, W.E., TRUEB, L. Biology of Amphibians. New York: McGraw-Hill, 670p, 1994.

18. FROST, D.R. Amphibians Species of the World: an on Line Reference.V2.21 Eletronic database available at http:// research.amnh.org/herpetology/amphibia/ index.html, (Acesso em abril de 2006) 2004.

19. GARCIA, P.C.A. Nova espécie de Eleutherodactylus Duméril \& Bibron, 1891, do estado de Santa Catarina, Brasil (Amphibia, Anura, Leptodactylidae). Biociências, v.2, p.5768, 1996.

20. GREEN, D.M., BOGART, J.P., ANTHONY, E.H., GENNER, D.L. An interactive, microcomputer-based karyotype analysis System for phylogenetic cytotaxonomy. Comput. Biol. Med., v.10, p.219-227, 1980.

21. HEYER, W.R. Variation, systematics, and zoogeography of Eleutherodactylus guentheri and closely related species (Amphibia: Anura: Leptodactylidae). Smith. Contrib. Zool., v.402, p.1-42, 1984.

22. HEYER, W.R. New species of frogs from Boracéia, São Paulo, Brazil. Proceedings of the Biological Society of Washington, v.98, p.657-671, 1985.

23. KAISER, H., GREEN, D.M., SCHMID, M. Systematics and biogeography of Eastern Caribbean frogs (Leptodactylidae: Eleutherodactylus), with the description of a new species from Dominica. Canadian Journal of Zoology, v.72, p.2217-2237, 1994.

24. KAISER, H., DWYER, C.M., FEITCHINGER, W., SCHMID, M. A new species of Eleutherodactylus (Anura: Leptodactylidae) from Tobago, West Indies and its morphometric and cytogenetic characterization. Herpetological Natural History, v.3, p.151-163, 1995.

25. KING, M. Amphibia. In: JOHN, B. (Ed.) Animal Cytogenetics. Amphibia, 4. Chordata 2. Berlin: Gebruder Borntraeger, 241p, 1990.

26. KURAMOTO, M. A list of chromosome numbers of anuran Amphibians. Bull. Fukuoka Univ. Educ., v.39, p.83-127, 1990.

27. LANGONE, J.A., SEGALLA, M.V. Una nueva especie de Eleutherodactylus del estado de Parana, Brasil. Com. Zool. Mus. Hist. Nat. v.12, p.1-8, 1996.

28. LEÓN, P.E. Report of the chromosome numbers of some Costa Rica anurans. Ver. Biol. Trop., v.17, p.119-124, 1970.

29. LYNCH, J.D. The species groups of the South American frogs of the genus Eleutherodactylus (Leptodactylidae). Ocas. Pap. Mus. Nat. Hist. Univ. Kan., v.61, p.1-24, 1976.

30. LYNCH, J.D., DUELLMAN, W.E. Frogs of the genus Eleutherodactylus in Western Ecuador: systematics, ecology, and biogeography. Mus. Nat. Hist. Univ. Kans. Spec. Publ., v.23, p.1-236, 1997.

31. LUCCA, E.J., JIM, J. Os cromossomos de alguns Leptodactylidae (Amphibia-Anura). Rev. Brasil. Biol., v.34, p.407-410, 1974.

32. LUCCA, E.J., JIM, J., FORESTI, F. Chromosomal studies in twelve species of Leptodactylidae and one Brachycephalidae. Caryologia, v.27, p.183-192, 1974.

33. MIYAMOTO, M.M. Frogs of the Eleutherodactylus rugulosus group: a cladistic study of allozyme, morphological and karyological data. Syst. Zool., v.32, p.109-124, 1983.

34. MIYAMOTO, M.M. Central American frogs allied to Eleutherodactylus cruentus: allozyme and morphological data. J. Herpetol., v.18, p.256-263, 1984.

35. PADIAL, J.M., De La RIVA, I. Rediscovery, redescription, 
and advertisement call of Eleutherodactylus heterodactylus (Miranda-Ribeiro, 1937) (Anura: Leptodactylidae), and notes on other Eleutherodactylus. J. Herpetol., v.39, p.372-379, 2005.

36. SAVAGE, J.M., MYERS, C.W. Frogs of the Eleutherodactylus biporcatus group (Leptodactylidae) of Central America and Northern South America, including rediscovered, ressucted and new taxa. Am. Mus. Novit. v.3357, p.1-48, 2002.

37. SAVAGE, J.M., De WEESE, J.E. A new species of Leptodactylidae frog, genus Eleutherodactylus, from the Cordillera de Talamanca, Costa Rica. Bull. Southern California Acad. Sci., v.78, p.107-115, 1979.

38. SAVAGE, J.M., De WEESE, J.E. The status of the Central American leptodactylid frogs Eleutherodactylus melanostictus (Cope) and Eleutherodactylus platyrhyncus (Günther). Proc. Biol. Soc. Wash., v.93, p.928-942, 1980.

39. SCHMID, M., STEINLEIN, C., KAUSCH, K., HAAF, T. Sex-determinating mechanisms and sex chromosomes in Amphibia. In: GREEN, D.M., SESSIONS, S.K.(Eds.) Amphibian Cytogenetics and Evolution. San Diego: Academic Press, p. 393-430, 1991.

40. SCHMID, M., STEINLEIN, C., FEICHTINGER, W. Chromosome banding in Amphibia XVII. First demonstration of multiple sex chromosome in amphibian: Eleutherodactylus maussi (Anura, Leptodactylidae). Chromosoma, v.1001, p.284292, 1992.

41. SCHMID, M., FEICHTINGER, W., STEINLEIN, C., RUPPRECHT, A., HAAF, T., KAISER, H. Chromosome banding in Amphibia XXIII. Giant W sex chromosomes and extremely small genomes in Eleutherodactylus euphronides and Eleutherodactylus shrevei (Anura, Leptodactylidae). Cytogenet. Genome Res., v.97, p.81-94, 2002a.
42. SCHMID, M., HAAF, T., STEINLEIN, C., NANDA, I., MAHONY, M. Chromosome banding in Amphibia XXV. Karyotype evolution and heterochromatin characterization in Australian Mixophyes (Anura, Myobatrachidae). Cytogenet. Genome Res., v.97, p.239-253, 2002b.

43. SCHMID, M., FEICHTINGER, W., STEINLEIN, C., HAAF, T., SCHARTL, M., VISBAL GARCÍA, R., MANZANILLA PUPO, J., FERNÁNDEZ BADILLO, A. Chromosome banding in Amphibia XXVI. Coexistence of homomorphic XY sex chromosomes and a derived Y-autosome translocation in Eleutherodactylus maussi (Anura, Leptodactylidae). Cytogenet. Genome Res., v.99, p.330-343, 2002c.

44. SCHMID, M., FEICHTINGER, W., STEINLEIN, C., VISBAL GARCIA, R., FERNÁNDEZ BADILLO, A. Chromosome banding in Amphibia XXVIII. Homomorphic XY sex chromosomes and a derived $\mathrm{Y}$ autosome translocation in Eleutherodactylus riveroi (Anura, Leptodactylidae). Cytogenet. Genome Res., v.101, p.62-73, 2003.

45. SIQUEIRA Jr, S., ANANIAS, F., RECCO-PIMENTEL, S.M. Cytogenetics of three Brazilian species of Eleutherodactylus (Anura, Leptodactylidae) with 22 chromosomes and re-analysis of multiple translocations in E. binotatus. Genetics and Molecular Biology, v.27, p.363-372, 2004.

46. SIQUEIRA Jr, S., AGUIAR Jr, O. SOUZA, M.B., RECCOPIMENTEL, S.M. Variação cromossômica intra-individual em Eleutherodactylus fenestratus (Leptodactylidae, Eleutherodactylinae). CD Resumos do $51^{\circ}$ Congresso Brasileiro de Genética, Águas de Lindóia, SP, p. 399, 2005.

47. SOUZA, M.B. Diversidade de anfíbios nas unidades de conservação ambiental: Reserva Extrativista do Alto Juruá (REAJ) e Parque Nacional da Serra do Divisor (PNSD), AcreBrasil. Tese de Doutorado, Instituto de Biociências, UNESP, Rio Claro, SP, 2003. 